\title{
PRESERVAÇÃO DA FÍSTULA ARTERIOVENOSA: AÇÕES CONJ UNTAS ENTRE ENFERMAGEM E CLIENTE
}

\author{
Preservation of arteriovenous fistula: conjunct actions from nursing and client \\ Preservación de la fistula arteriovenosa: acciones conjuntas de la enfermería y cliente
}

Alessandra Guimarães Monteiro Moreira ${ }^{1}$

Sílvia Teresa Carvalho de Araújo ${ }^{2}$

Thalita Souza Torchi ${ }^{3}$

\section{RESUMO}

0 objetivo deste estudo foi identificar os cuidados dos clientes em tratamento hemodialítico com o local da punção para a preservação da fístula arteriovenosa. Estudo qualitativo, descritivo-exploratório, que incluiu 17 clientes do Hospital Universitário (RJ) com doença renal crônica de qualquer etiologia, maiores de 18 anos de idade, de ambos os sexos, tendo como acesso a fístula arteriovenosa há, no mínimo, seis meses. Como resultados, obteve-se que $100 \%$ dos entrevistados conhecem a necessidade de cuidar e manter a via de punção pérvia; $42 \%$ já tiveram algum tipo de intercorrência na fístula, destacando-se a dor no membro e a perda do frêmito; $29 \%$ realizam compressa de gelo em casa, após a hemodiálise; $17 \%$ afirmaram que, a qualquer acontecimento diferente ou anormal, conversam com a equipe médica e de enfermagem. Concluiu-se que a enfermeira que orienta o cliente para a preservação do acesso necessita perceber indícios não verbais que apontem fragilidades no desempenho de papéis para 0 autocuidado.

Palavras-chave: Enfermagem. Comunicação. Diálise renal. Fístula arteriovenosa.

\begin{abstract}
Identify the customer care on haemodialysis treatment with the puncture site for the preservation of arteriovenous fistula. Method: A qualitative study, descriptive-exploratory which included 17 clients of the University Hospital (RJ) with chronic kidney disease of any etiology, greater than 18 years old, both sexes, having as access an arteriovenous fistula for at least six months. Results: $100 \%$ of the interviewees know the necessity of caring and maintain the via puncture pervious, $42 \%$ had some type of intercadent in the fistula, stand out the pain in the limb and loss in the thrill, 29\% carry ice pack at home after haemodialysis. And $17 \%$ assert that any different or abnormal event they talk to the medical staff and nursing. Conclusion: A nurse who directs the client to the preservation of access needs to realize nonverbal clues that point to weaknesses in the performance roles for self-care.
\end{abstract}

Keywords: Nursing. Communication. Renal Dialysis. Arteriovenous Fistula.

\section{Resumen}

identificar cuáles son los cuidados que reciben los clientes en tratamiento de hemodiálisis con local de punción para la preservación de la fistula arteriovenosa. Método: estudio cualitativo, descriptivo-exploratorio, que incluye 17 clientes del Hospital Universitario (RJ) con enfermedad renal crónica de cualquier etiología, mayores de 18 años de edad, de ambos sexos, teniendo como acceso la fistula arteriovenosa por lo mínimo seis meses. Resultados: el $100 \%$ de los entrevistados conocen la necesidad de cuidar y mantener la vía de punción permeable; $42 \%$ ya tuvieron algún tipo de problema con la fistula, destacándose el dolor en el miembro y pierda del frémito; $29 \%$ realizan compresa de hielo en casa, después de la hemodiálisis. $17 \%$ afirmaron que cualquier acontecimiento diferente de lo esperado, lo informaban al equipo médico y de enfermería. Conclusión: la enfermería que orienta el cliente para la preservación del acceso necesita enterarse de indicios no verbales que indiquen fragilidades en el desempeño de los papeles para el autocuidado.

Palabras-clave: Enfermería. Comunicación. Diálisis Renal. Fístula arteriovenosa.

'Aluna do Curso de Mestrado em Enfermagem pela Escola de Enfermagem Anna Nery, da Universidade Federal do Rio de Janeiro (UFRJ), Enfermeira. Membro do grupo de pesquisa Comunicação em Enfermagem Hospitalar - Cliente de Alta Complexidade, EEAN/UFRJ. Rio de Janeiro - RJ. Brasil. E-mail: Alessandra.moreira52@yahoo.com.br; ${ }^{2}$ Doutora em Enfermagem. Professora Associada II do Departamento de Enfermagem Médico-Cirúrgica (DEMC), da Escola de Enfermagem Anna Nery, da Universidade Federal do Rio de Janeiro. Doutora em Enfermagem. Líder do grupo de pesquisa Comunicação em Enfermagem Hospitalar - Cliente de Alta Complexidade, EEAN/UFRJ. Rio de Janeiro - RJ. Brasil. E-mail stcaraujo@gmail.com; ${ }^{3}$ aluna do Curso de Mestrado da Escola de Enfermagem Anna Nery (EEAN), da Universidade Federal do Rio de Janeiro. Enfermeira. Especialista em Enfermagem em Nefrologia. Membro do grupo de pesquisa Comunicação em Enfermagem Hospitalar - Cliente de Alta Complexidade, EEAN/UFRJ. Rio de Janeiro - RJ. Brasil. E-mail: nurtha@yahoo.com.br. 


\section{INTRODUÇÃO}

0 presente estudo foi desenvolvido durante o cuidado de enfermagem na terapia substitutiva renal (TSR), em sessão de hemodiálise (HD), junto aos clientes com acesso vascular através de fístula arteriovenosa (FAV), e buscou demonstrar o que eles conhecem e fazem para a preservação do acesso vascular.

0 interesse em estudar essa temática emergiu pela necessidade de intervenção, especialmente pelas dúvidas, ainda presentes, sobre alguns cuidados prestados a esta clientela. Assim, o conhecimento, a partir de uma investigação científica, foi o auxílio para compreender a complexidade das intervenções e suprir as demandas implícitas neste cuidado.

A inserção precoce na área de nefrologia contribuiu para a formação acadêmico-profissional sobre como se dá o cuidado de enfermagem, o próprio autocuidado do cliente com o acesso e o levantamento de quais são os problemas mais comuns que influenciam na preservação da FAV. Sendo assim, para este estudo foi elaborado o seguinte objetivo: Identificar quais os cuidados dos clientes com o local da punção para a preservação da fístula arteriovenosa.

0 desenvolvimento de pesquisas em enfermagem é fundamental, pois permite a construção do conhecimento próprio, possibilitando prestar melhor assistência de enfermagem ao cliente, embasada em conhecimento científico, propiciando enriquecimento no campo prático e profissional, bem como possibilita a busca de soluções para os problemas vivenciados no cotidiano ${ }^{1}$.

Desse modo, o enfermeiro que possui uma visão holística do cuidado de enfermagem com a FAV, ao realizar os procedimentos de punção e retirada da agulha, no corpo do cliente dependente da HD, poderá refletir sobre a melhor conduta e abordagem para preservá-la e evitar eventuais complicações. A proximidade do enfermeiro-cliente permite melhor compreensão sobre o plano de cuidado específico para cada cliente, atendendo de maneira individual e singular suas necessidades.

\section{REVISÃO DA LITERATURA}

A Doença Renal Crônica (DRC) é uma deterioração progressiva e irreversível da função renal, na qual a capacidade do corpo para manter a homeostasia metabólica e hidroeletrolítica falha, resultando em uremia, que é definida pela retenção de ureia e outros produtos nitrogenados no sangue ${ }^{2}$.

À medida que a função renal diminui, os produtos finais do metabolismo proteico, normalmente excretados através da urina, acumulam-se no sangue. A uremia desenvolve-se e afeta de maneira adversa todos os sistemas do corpo. Quanto maior o acúmulo de produtos de degradação, mais graves serão os sintomas da DRC 3 .

A Iniciativa de Qualidade em Resultados de Doenças Renais da Fundação Nacional do Rim dos Estados Unidos define DRC como a presença de dano renal ou diminuição da função renal por três ou mais meses, sendo classificada em estágios ${ }^{4}$. Inicialmente os casos que apresentam fatores de risco para doença renal variam do estágio I, cuja filtração glomerular é normal (e" $90 \mathrm{ml} / \mathrm{min}$ ), até o estágio IV, que é caracterizado por perda severa de função com filtração glomerular entre $15 \mathrm{e}$ $29 \mathrm{ml} / \mathrm{min}$.

A insuficiência renal terminal, estágio $V$, é caracterizada por filtração glomerular menor do que $15 \mathrm{ml} / \mathrm{min}$, havendo necessidade de diálise 4 , e é o resultado final de múltiplos sinais e sintomas decorrentes da incapacidade renal de manter a homeostasia interna do organismo. Uma vez instalada, é necessário um tratamento contínuo para substituir a função renal. Os tratamentos disponíveis são a diálise peritoneal, hemodiálise e o transplante renal ${ }^{3}$.

A hemodiálise tem como objetivo extrair as substâncias nitrogenadas tóxicas do sangue e remover 0 excesso de água; é realizada por meio de um sistema extracorpóreo, no qual o sangue flui através de um acesso vascular, sendo a FAV uma das modalidades de acesso, dentro das quatro existentes ${ }^{4}$. Os tipos disponíveis são: cateter duplo lúmen (podendo ser inseridos em veia subclávia, jugular interna e femoral), a FAV, prótese de politetrafluoretileno (PTFE) e shunt?.

0 fluido sanguíneo é impulsionado por uma máquina para o sistema de circulação extracorpórea até o dialisador, onde ocorrem as trocas entre o sangue e o banho de diálise ou dialisato. A difusão de solutos entre o sangue e a solução de diálise resulta na remoção de escórias metabólicas ${ }^{5}$, assim o sangue é filtrado e, em seguida, devolvido ao cliente; esse processo só pode ser estabelecido através do acesso vascular.

A fístula é conhecida como o acesso permanente, criado por meio cirúrgico (usualmente no antebraço) ao unir a artéria a uma veia. 0 ramo venoso da FAV dilata-se e sua parede se torna mais espessa, permitindo repetidas punções. As agulhas são inseridas no vaso com finalidade de obter o fluxo sanguíneo adequado para passar através do dialisador ${ }^{2}$.

A hemodiálise é uma forma indefinida de prolongar a vida, visto que não altera a evolução natural da doença renal subjacente, nem substitui por completo a função renal; por isso, o cliente está sujeito a inúmeros problemas e complicações, entre os quais se destaca a coagulação aumentada pelo dialisador, que pode ser evitada pelo ajuste das doses de heparina ${ }^{6}$.

Durante a diálise, o paciente, o dialisador e 0 dialisato precisam de constante monitoração e avaliação. Para evitar as complicações como embolia gasosa, 
ultrafiltração inadequada ou excessiva (hipotensão, câimbra, vômitos), extravasamentos sanguíneos e contaminações no acesso, a enfermeira realiza não só essa vigilância, mas também desempenha um papel importante na educação do cliente 7 .

Preparar o cliente para a hemodiálise é um desafio para o enfermeiro, pois o cliente pode não compreender o impacto da diálise, e as necessidades de aprendizado podem passar despercebidas. A boa comunicação entre a equipe de diálise, equipe da unidade e o cliente é essencial para fornecimento do cuidado adequado e contínuo, visando estimular o autocuidado 3 .

0 diagnóstico e o tratamento têm impacto muito grande sobre o cliente e a família. Sendo assim, o enfermeiro deve estabelecer uma relação de confiança, através da qual o cliente se sinta encorajado a questionar, tirar suas dúvidas e aprender como evitar as complicações, e não ser passivo no tratamento, atentando para as alteraç̧ões físicas e fisiológicas que ocorrem durante a evolução da DRC.

0 autocuidado tem como propósito as ações, que, seguindo um modelo de recomendações, contribuem de maneira específica na integridade, no equilíbrio das funções orgânicas e na preservação do acesso vascular. Portanto, essa prática constitui habilidade humana que significa cuidar de si mesmo ${ }^{8} \cdot A$ comunicação efetiva entre cliente e equipe de enfermagem é essencial para o incentivo ao desempenho de papéis para o autocuidado.

No ato de cuidar, a enfermagem, como ciência, implica o estabelecimento de interação entre os sujeitos (quem cuida e quem é cuidado), de forma que, além de uma ação técnica, ocorre uma ação sensível com respeito e carinho aos pacientes. " 0 cuidado deve ser sentido, vivido. E, para que o cuidado seja integrado no nosso dia-a-dia, é preciso absorvê-lo, permitir que ele faça parte de nós mesmos, transformá-lo em estilo de vida"

0 vínculo existente entre a equipe de enfermagem e o cliente torna-se intenso, pois o tratamento hemodialítico é contínuo. Este vínculo às vezes se torna facilitador para a assistência quando ampliamos nossa capacidade de observação, tanto para os aspectos objetivos relativos ao procedimento técnico, como para os aspectos subjetivos da clientela relativos à singularidade da comunicação corporal. 0 profissional de enfermagem ao desenvolver, continuamente, habilidades técnicas e humanas para intervir, deverá também desenvolver e aprimorar sua capacidade de se comunicar ${ }^{10}$.

Podemos aguçar nossa capacidade de captar as necessidades de um cuidado efetivo por meio das diferentes formas de se comunicar. Através da leitura das manifestações não verbais da clientela, podemos criar maneiras diferentes de intervir, a fim de facilitar e alcançar os objetivos para uma assistência de enfermagem mais singular, individualizada e solidária à clientela ${ }^{11}$.

\section{MÉTODO}

Estudo de abordagem qualitativa com finalidade descritiva e exploratória. 0 projeto recebeu aprovação pelo Comitê de Ética da Escola de Enfermagem Anna Nery e Hospital São Francisco de Assis da Universidade Federal do Rio de Janeiro; foi protocolado sob o número 006/2010 e desenvolvido no setor de hemodiálise de um Hospital Universitário (RJ), no período de janeiro a dezembro de 2011.

Para a caracterização da clientela foi realizado um levantamento de dados sobre o perfil sociodemográfico com sete variáveis: idade, gênero, estado civil, profissão, escolaridade, tempo de tratamento hemodialítico e as doenças crônicas dos participantes do estudo. Estes dados foram obtidos com o cliente na sala de espera, o que possibilitou conhecer melhor os sujeitos envolvidos na pesquisa.

Foi utilizada também a técnica de observação não participante do cliente durante a punção e a entrevista gravada após sessão de hemodiálise, com questões semiestruturadas sobre os cuidados dos clientes para preservação da FAV.

Participaram do estudo 17 clientes cadastrados no hospital e atendidos no setor de nefrologia, que atenderam os seguintes critérios de inclusão: ser adulto maior de 18 anos de idade, com doença renal crônica de qualquer etiologia, de ambos os sexos; encontrar-se em programa de hemodiálise, com acesso através de FAV, há um período mínimo de seis meses; e não apresentar sinais clínicos de desconforto no dia da coleta de dados.

Para assegurar o anonimato utilizamos pseudônimos para identificar os sujeitos. Para cada paciente foi dada a opção de escolher um símbolo da natureza; os símbolos a seguir foram os escolhidos: Sol, Lua, Vento, Água, Brisa, Flor e Terra.

Os depoimentos transcritos permitiram a emergência de categorias por sua relevância teórica e repetição ${ }^{12}$ utilizando a técnica de tratamento dos dados qualitativos com a análise de conteúdo ${ }^{13}$. Foram apontadas as características dos participantes e triangulados os dados produzidos nas duas técnicas para descrever os tipos de ações realizadas por eles durante a sessão de hemodiálise e aquelas descritas como sendo realizadas no domicílio.

\section{RESULTADOS E DISCUSSÕES}

A caracterização dos sujeitos participantes quanto a idade, gênero, estado civil, profissão, escolaridade, tempo de tratamento hemodialítico e outras doenças crônicas permitiram conhecer melhor a clientela assistida nas sessões de hemodiálise, conforme a Tabela 1. 
Tabela 1: Distribuição dos clientes em tratamento hemodialítico, segundo idade, gênero, estado civil, profissão eescolaridade. Rio de Janeiro, 2011. ( $N=17)$.

\begin{tabular}{|c|c|c|}
\hline Característica & $\mathbf{N}$ & $\%$ \\
\hline \multicolumn{3}{|l|}{ Idade } \\
\hline $30-39$ & 2 & 12 \\
\hline $40-49$ & 6 & 35 \\
\hline $50-59$ & 8 & 47 \\
\hline $60-69$ & 1 & 6 \\
\hline \multicolumn{3}{|l|}{ Gênero } \\
\hline Feminino & 9 & 53 \\
\hline Masculino & 8 & 47 \\
\hline \multicolumn{3}{|l|}{ Estado civil } \\
\hline Solteiro & 2 & 12 \\
\hline Casado & 11 & 65 \\
\hline Separado/Divorciado & 4 & 23 \\
\hline \multicolumn{3}{|l|}{ Profissão } \\
\hline Dolar & 8 & 47 \\
\hline Pedreiro & 2 & 12 \\
\hline Costureira & 1 & 6 \\
\hline Aposentado & 6 & 35 \\
\hline \multicolumn{3}{|l|}{ Escolaridade } \\
\hline $1^{\circ} \mathrm{grau}$ incompleto & 13 & 76 \\
\hline $1^{10}$ grau completo & 3 & 18 \\
\hline $2^{\circ}$ grau completo & 1 & 6 \\
\hline
\end{tabular}

A Tabela 1 mostra que a faixa etária com maior número de sujeitos é de 40 a 59 anos. Este dado denota que as alterações inerentes ao processo de envelhecimento não significam maior suscetibilidade à doença, mas a probabilidade de seu aparecimento aumenta com a idade, uma vez que o envelhecimento torna as pessoas mais vulneráveis aos processos patológicos. Dados da pesquisa realizada pela sociedade Brasileira de Nefrologia (2010) mostram que a faixa etária na qual mais se evidenciam casos de insuficiência renal é de 19 a 64 anos $(67,7 \%)^{14}$. Estudos populacionais em diferentes países têm demonstrado prevalência da Doença Renal Crônica (DCR) de 7,2\% para indivíduos acima de 30 anos e $28 \%$ a $46 \%$ em indivíduos acima de 64 anos.

Houve uma predominância do sexo feminino entre os participantes do estudo; contudo, deve-se ressaltar que a participação na pesquisa foi de livre escolha e os pacientes do sexo feminino estiveram mais abertos ao diálogo e à participação. Entretanto, dados da Sociedade Brasileira de Nefrologia ${ }^{14}$ demonstram que o percentual de pacientes do sexo masculino que atualmente realizam diálise corresponde a $57 \%$.
Observou-se que $65 \%$ dos sujeitos eram casados ou tinham um companheiro. Os principais relatos dos entrevistados quanto à importância de ter uma relação estável indicaram a participação efetiva dos companheiros nas atividades diárias e no apoio ao tratamento hemodialítico.

Embora um dos cuidados para a preservação da FAV seja o de não fazer esforço físico com o braço com a FAV, notamos que dois dos sujeitos tinham trabalhos que exigiam movimentos bruscos, repetidos e de força física com os membros superiores.

0 número de sujeitos com baixo grau de escolaridade foi significativo (76\%), o que retrata, sem dúvida, a situação nacional em termos de acesso à educação. Em outras palavras, ainda é preocupante o nível cultural e socioeconômico da população brasileira, ou seja, com restrito acesso ao ensino fundamental, o que acarreta dificuldade no nível de informação ${ }^{15}$. Desta forma, identificamos a necessidade em criar estratégias de comunicação para orientar estes clientes quanto aos cuidados. Cabe à equipe de enfermagem informar e educar quanto às necessidades do cuidar no tratamento de hemodiálise. 
Tabela 2: Distribuição dos clientes em tratamento hemodialítico, segundo o tempo de tratamento e principais doenças crônicas associadas, Rio de Janeiro, 2011. ( $N=17)$.

\begin{tabular}{llc}
\hline Características & N & \% \\
\hline Tempo de tratamento & 1 & 6 \\
$2-3$ & 3 & 18 \\
$4-5$ & 5 & 29 \\
$6-7$ & 5 & 29 \\
$8-9$ & 1 & 6 \\
$10-11$ & 2 & 12 \\
$12-13$ & & \\
Doenças crônicas & 8 & 47 \\
Hipertensão arterial & 4 & 23 \\
Indeterminada & 3 & 18 \\
Diabetes melito & 2 & 12 \\
\hline Nefroesclerose hipertensiva & & \\
\hline
\end{tabular}

Os dados da Tabela 2 demonstram uma variação de 4 a 9 anos no tempo de tratamento de HD destes clientes. Embora a HD possa prolongar a vida, ela não altera a evolução natural da doença renal subjacente, e nem substitui por completo a função renal. A doença renal é considerada um grande problema de saúde pública, porque causa elevada taxa de morbidade e mortalidade e, além disso, tem impacto negativo sobre a qualidade de vida relacionada à saúde. Estudos recentes nos Estados Unidos estimam que 26 milhões de adultos sofram da doença, que aumenta as chances de morte prematura em virtude da propensão desses clientes ao desenvolvimento de doenças cardiovasculares ${ }^{5}$.

Os principais fatores de risco para doença renal crônica são: diabetes, hipertensão arterial sistêmica, problemas circulatórios e cardíacos, histórico anterior familiar e idade acima de 65 anos $^{5}$. 0 diabetes melito e a hipertensão arterial são doenças silenciosas, pois nem sempre apresentam sinais e sintomas clássicos, por isso muitos desconhecem que as têm. Outros têm conhecimento delas, porém não aderem ao tratamento por julgarem-no desnecessário. Desta forma, desenvolvem lesões lentas e progressivas nos órgãos, dentre eles os rins, o que pode levar à insuficiência renal crônica.

Durante o tratamento, o cliente em HD necessita manter outros cuidados, tais como vigiar o funcionamento do acesso por meio da palpação e percepção do frêmito; observar sinais e sintomas de infecção; realizar a higiene; não permitir punções venosas por outros profissionais para administração de medicamentos; verificar a pressão arterial no braço da fístula; evitar qualquer compressão e dormir sobre o braço do acess $0^{16}$.

0 cliente de HD é orientado pela equipe de enfermagem de forma contínua e direta a cuidar de sua fístula. 0 cliente deve estar apto a compreender o funcionamento de seu acesso vascular e o objetivo das medidas de precaução para evitar sua inoperância. Esses cuidados destinam-se a proteger o acesso do excesso de peso sobre o braço, pois poderá interromper o fluxo sanguíneo, ocasionando trombose no acesso venoso ${ }^{16}$.

Dos clientes entrevistados $100 \%$ relataram que conhecem a necessidade de cuidar e manter a via de punção pérvia. Embora um dos cuidados para a preservação da FAV seja o de não fazer esforço físico com o braço afetado, observamos que dois dos sujeitos executavam atividade laboral que exigia movimentos bruscos, repetidos e de força física com os membros superiores.

Um dos cuidados mais citados pelos entrevistados foi o de não pegar peso.

\section{[...] eu não pego peso no braço desde que fiz a operação, tomo todo o cuidado [...] (Sol).}

Os dados demonstraram que $42 \%$ dos participantes já tiveram algum tipo de intercorrência na fístula:

[...] tem vez que sai muito sangue quando tira a agulha, fico apertando bastante tempo para não me sujar, outro dia foi sangue pra todo lado [...] (Terra).

[...] outro dia meu neto sem querer me deu uma cabeçada no braço da fístula e quando fui ver tinha parado, fiquei muito triste..., mas agora fiz outra, e essa tá boa [...] (Lua).

Dentre as ocorrências mais citadas destacou-se a dor no membro da FAV e perda do frêmito.

[...] sinto dor no braço, algumas vezes, depois de fazer a hemodiálise, mas quando chego em casa, 
coloco gelo e depois de um tempo passa. [...] (Brisa).

\section{[...] meu susto foi muito grande quando acordeie vi que tinha algo errado na minha FAV, ela não tava mais tremendo, não sentia o sangue passar, ... Chorei porque sabia que iria penar de novo para fazer outra [...] (Vento).}

0 cliente informou que a ocorrência descrita se deu enquanto dormia. Quando acordou no dia seguinte, depois de uma noite de sono dormindo sobre a FAV, havia perdido o frêmito.

Dos entrevistados, $29 \%$ relataram colocar compressa de gelo em casa, após fazer a hemodiálise; $17 \%$ afirmaram que sempre que acontece algo de diferente ou anormal, conversam com a equipe médica e de enfermagem.

[...] toda vez que tem algo de estranho falo logo com a enfermeira ou o médico, procuro me cuidar, não quero que as coisas piorem [...] (Vento).

Foram observadas as ações de cuidado de enfermagem desde a entrada do cliente no setor; como a observação da lavagem da FAV com água e sabão pelo cliente; a verificação do peso e pressão arterial; o uso de EPI (equipamento de proteção individual) pelo enfermeiro; a antissepsia no local da punção; 0 exame físico da fístula, para identificar alguma alteração, até 0 momento de o cliente ser conectado ao hemodialisador. Nessa observação, somente alguns clientes mostraram-se pró-ativos.

Entretanto, o que sobressaiu foi a forma como o profissional se dirigia e se comunicava com o cliente, imprimindo mais ou menos atenção e carinho. A interação, estabelecida com o cliente no ato de cuidar, emergiu do tipo de comunicação estabelecida entre o enfermeiro e o cliente.

No ato da punção foram identificadas diferentes formas de interação entre cliente e profissional, acarretando diferentes formas de cuidar. Para a realização da punção é necessário que haja avaliação prévia e escolha do melhor local para a punção pelo profissional de enfermagem; isto denota um cuidado com o cliente. Além disso, os sentidos corporais captam as maiores mensagens que são transmitidas pela linguagem corporal; quando não são consideradas no cuidado de maneira devida, não há comunicação efetiva.

A comunicação, portanto, é um alicerce importante para que a relação de cuidado se estabeleça de forma efetiva $e$ eficaz. Por meio dela podemos identificar formas de diminuir os possíveis danos causados ao cliente. A comunicação não verbal deve ser utilizada para perceber com maior precisão os sentimentos do cliente, suas dúvidas e dificuldades de verbalização.

Diante do exposto, não é possível afirmar que as manifestações que podem ser percebidas durante as observações no campo foram somente as reveladas e manifestadas nesta pesquisa, visto que somente estudos mais complexos de comunicação seriam capazes de desvelar o que realmente se passa em cada atitude do ser humano ${ }^{17}$.

Identificou-se que o cuidar em hemodiálise vai além do conhecimento técnico e científico, mesmo que esse processo envolva complexidade e especificidade para os clientes; ser cuidado significou estabelecer relacionamento interpessoal. As interações no cuidado não podem ser estabelecidas de maneira puramente técnica. A utilização dos órgãos dos sentidos não restringe a assistência de enfermagem ao aspecto racionalizado, puramente instrumental. É preciso intensificar a afetividade para não empobrecer a interaçãa ${ }^{17}$.

\section{CONCLUSÃO}

0 tratamento hemodialítico é contínuo e sustenta a vida do paciente; embora haja a rotina, o enfermeiro e o cliente a cada dia estabelecem uma forma de comunicação diferente. É a partir da compreensão da comunicação estabelecida que a assistência à clientela torna-se eficaz, pois, se não houver esta percepção, as ações serão puramente tecnicistas sem perspectiva do cuidado humanizado.

0 estudo mostrou que os clientes afirmam conhecer os cuidados para a preservação da FAV, porém nem todos os cuidados extensivos ao domićlio são seguidos pela maioria deles. Existe o envolvimento da enfermagem com orientações de cuidados para a preservação da FAV dentro desse ambiente hospitalar, porém com participação passiva dos clientes.

A relação estreita entre enfermagem e cliente renal crônico dentro do setor de hemodiálise é grande diferencial que pode favorecer a comunicação como artíício do cuidado de enfermagem, que contribuirá para o binômio cuidado de enfermagem e autocuidado da clientela.

A comunicação é meta para o cuidado e deve ser pontual para atender às necessidades implíitas nas expressões do corpo do cliente, principalmente aquelas faciais e de movimento durante a sessão de hemodiálise, indicativas de limitações para enfrentar, cuidar e preservar a fístula.

Por meio do atendimento às necessidades implíitas nessa comunicação, o enfermeiro amplia o respeito e a consideração do cliente e reforça a abordagem terapêutica na interação, para promover junto a ele o papel essencial proativo para o autocuidado na preservação da fístula durante todo o tratamento.

\section{REFERÊNCIAS}

1. Cassiani SHB, Passarelli LR. Pesquisar em enfermagem: um processo de ação de enfermagem. Rev. gaúch. enferm. 1999 jan. 20(1): 91-110.

2. Smeltzer SC, Bare BG. Brunner \& Suddarth: Tratado de Enfermagem Médico Cirúrgica. 10. ed. Rio de Janeiro: Guanabara Koogan; 2005. 
3. Barbosa DA. et al. Co-morbidade e mortalidade de pacientes em início de diálise. Acta paul. enferm. 2006 jul./set. 16(3): 304-9.

4. The National Kidney Foundation Kidney Disease Outcomes Quality Initiative. Are You at Increased Risk for Cronic Kidney Disease? 2010; [citado em 2012 jan. 28]. Disponível em: <http://www.kidney.org/professionals/ kdoqi/>.

5. Junior MAG, Andreoli MCC, Sardenberg C, Santos BFC, Neto MC. Diálise no paciente com insuficiência renal crônica: hemodiálise e diálise peritoneal. In: Barros, E, Manfro RC, Gonçalves LFS. Nefrologia: rotinas, diagnóstico e tratamento. Porto Alegre: ARTMED, 2006.

6. Eschbach JW, Adamson JW. Guidelinesfor recombinant human erythopoirtin therapy. Am J Kidney Dis. 1989 ago. 14(2 supp;1): 2-8.

7. Arone EM, Philippi MLS. Enfermagem Médico-cirúrgica aplicada ao sistema renal e urinário. 2. ed. e ampl. São Paulo: Editora Senac; 2005.

8. Orem DE. Enfermagem: conceitos de prática (1980). Traduzido por Fernando Volkmer;1985.

9. Waldow VR, Lopes MJM, Meyer DE. Maneiras de cuidar e maneiras de ensinar: a enfermagem entre a escola e a prática profissional. Porto Alegre: Artmed; 1995.

10. Marins AC, Araújo STC. A percepção auditiva da paralinguagem do paciente após tireoidectomia subtotal: ensino e pesquisa em enfermagem. Esc. Anna Nery Rev. Enferm. 2005 dez. 10(3): 586-91.

11. Koeppe GBO, AraújoSTC. Comunicação como temática de pesquisa na Nefrologia: subsídio para o cuidado de enfermagem. Acta paul. enferm. 2009. 22(especial 1)

12. Lakatos EM, Marconi MA. Metodologia do trabalho científico. 4. ed. São Paulo: Atlas, 1995.

13. Bardim L. Análise de conteúdo. Lisboa: Edições 70, 2007.

14. Sociedade Brasileira de Nefrologia. Censo de Diálise SBN 2010 [citado em 2012 dez. 12]. Disponível em: <http://www.sbn.org.br/>.

15. Ferreira V, Andrade D, Santos CB, Neto MM. Infecção em pacientes com cateter temporário duplo-lúmen para a hemodiálise. Rev. panam. infectol. 2005 abr.jun.; 7(2): 16-21.

16. Furtado AM, Lima FET. Autocuidado dos pacientes portadores de insuficiência renal crônica com a fístula artério-venosa. Rev. gaúch. enferm. 2006 dez. 27(4): 532-8.

17. Araujo STC. Os sentidos corporais dos estudantes no aprendizado da comunicação não verbal do cliente na recepção pré-operatória: uma semiologia da expressão através da sociopoética [tese]. Rio de Janeiro: Universidade Federal do Rio de Janeiro; 2000. 